\title{
面向知识图谱的位置敏感嵌入模型
}

\author{
张似衡 ${ }^{1,2)}$, 张文生 ${ }^{1)^{*}}$ \\ 1) (中国科学院自动化研究所精密感知与控制中心 北京 100091) \\ 2) (中国科学院大学 北京 100049) \\ (zhangwenshengia@hotmail.com)
}

\begin{abstract}
摘 要: 知识图谱嵌人将离散的符号化实体和关系映射到连续的低维空间中, 使后续的任务(如推理、图谱补全等) 可以通过代数运算的方式进行. 大体而言, 知识图谱嵌人可以分成 2 类主流方法: 平移距离模型和语义匹配模型. 从 知识图谱的图结构出发, 可以分析平移距离模型的学习瓶颈, 进而得出结论, 即平移距离模型的缺点是没有区别对 待实体在头部和尾部 2 个位置所应有的不同语义. 据此，提出位置敏感的嵌人表示(location-sensitive embedding, LSE) 模型. 与现有模型不同的是, LSE 仅对头部实体进行由不同关系决定的映射, 并且将关系建模为一般化的线性变换而 不仅是平移. 理论分析的结果保证了 LSE 的表示能力，同时揭示了与其他模型之间的联系. 同时，为了提高模型的 训练和推理效率, 提出简化模型 $\mathrm{LSE}_{d}$, 将线性变换限制为对角阵. 在 4 个大规模通用知识图谱数据集上使用链接预 测任务进行测试. 实验结果表明, 所提出的模型达到了最高或与当前最先进模型持平的性能.
\end{abstract}

关键词: 知识图谱; 表示学习; 平移距离模型; 位置敏感嵌人

中图法分类号: TP391.41 DOI: 10.3724/SP.J.1089.2021.18558

\section{Location-Sensitive Embedding for Knowledge Graph Embedding}

\author{
Zhang Siheng ${ }^{1,2)}$ and Zhang Wensheng ${ }^{1 *^{*}}$ \\ 1) (Precise Sensing and Control Center, Institute of Automation, Chinese Academy of Sciences, Beijing 100091) \\ 2) (University of Chinese Academy of Sciences, Beijing 100049)
}

\begin{abstract}
Knowledge graph embedding maps the discrete symbolic entities and relations into continuous low-dimensional space, enabling the downstream task (e.g., inference, knowledge graph completion and etc.) to be carried on in an algebraic manner. Roughly speaking, there are two main-streams towards knowledge graph embedding, translational distance models and semantic matching models. The learning bottleneck of the former caused by special graph structures is analyzed. And it is found out that translational distance models are to be blamed for treating head and tail entities from a same viewpoint. So, a location-sensitive embedding (LSE) model is proposed. Unlike previous models, it just transforms the head entity with relational-specific mapping. And it models relation as a general linear transformation instead of a translational operator. Its representation capacity and the relationship to current models are theoretically analyzed. Also, to make it efficient,

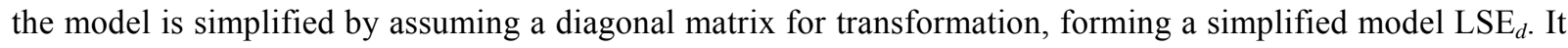
is evaluated that the proposed model on four large-scale knowledge graph datasets for link prediction task. Experiments have shown that the proposed model achieves highest, or at least competitive, performance compared with the state-of-the-art models.
\end{abstract}

收稿日期: 2020-08-06; 修回日期：2021-01-09. 基金项目：科技创新 2030一“新一代人工智能”重大项目(2018AAA0102100). 张似衡(1993-), 男, 博士研究生, 主要研究方向为机器学习、深度学习、知识图谱; 张文生(1965一), 男, 博士, 研究员, 博士生导师, CCF 会员, 论文通讯作者, 主要研究方向为人工智能、机器学习、大数据知识挖掘. 
Key words: knowledge graph; representation learning; translational distance model; location-sensitive embedding

随着大数据时代的到来, Google 于 2012 年提 出知识图谱改善用户搜索、内容聚合等服务, 掀起 了知识图谱研究的热潮 ${ }^{[1]}$. 知识图谱是一种描述实 体及其关系的结构化方式. 其中，实体被建模为节 点, 关系被建模为连接实体的不同类型的边, 通常 可以用三元组 $(h, r, t)$ 的形式表达.

尽管知识图谱具有良好的结构和定义，其潜 在的符号化特性却对自动构建和推理提出了挑战. 为了解决这个问题, 知识图谱嵌人将离散化的实 体和关系映射到连续的向量空间中，从而将关系 推理简化为代数运算 ${ }^{[2]}$. 知识图谱嵌人方法大致分 成 2 大类: 平移距离模型和语义匹配模型 ${ }^{[3]}$. 平移 距离模型将关系建模为平移变换, 使用距离评分 衡量三元组的正确性; 语义匹配模型使用相似度 评分. 此外, 还有一些研究引人了额外信息(如实 体类型、关系路径等)进行建模 ${ }^{[4]}$ ，这些方法在本文 的关注范围之外. 本文旨在通过分析平移距离模 型的缺点, 提出更加一般化的距离模型.

现有的平移距离模型对三元组所施加的约束 可以用一个一般化的公式 $f_{r}(\boldsymbol{h})+\boldsymbol{r}=f_{r}(\boldsymbol{t})$ 进行概 括. 其中, $f_{r}($.$) 是由关系决定的映射, 通常建模为$ 线性变换. 在 $T r a n s \mathrm{E}^{[5]}$ 模型中, $f_{r}($.$) 退化为恒等矩$ 阵, 即要求 $\boldsymbol{h}+\boldsymbol{r}=\boldsymbol{t}$. 这种简化忽略了实体在不同 关系下的不同语义，不能很好地处理“一对多”“多 对一”和 “多对多”的关系. 为了解决这个问题, TransR ${ }^{[6]}$ 引人由连接实体的关系所决定的变换矩 阵, 使 $f_{r}(\boldsymbol{x})=\boldsymbol{x} \boldsymbol{M}_{r}$; 随后, $\mathrm{TransH}^{[7]}$ 将变换矩阵固 定为投影矩阵，投影面的正规向量由关系决定; $\operatorname{TransA}^{[8]}$ 和 $T r a n s D^{[9]}$ 等模型对变换矩阵引人了新 约束, 大大减少了参数的数量.

然而，这些模型并没有实质上的提高. 从实验 结果上分析, 经过仔细训练的 TransE 模型的性能 并不低于后来的改进模型 ${ }^{[10]}$. 从理论上分析，平 移距离模型不能建模环状结构的图谱. 已有工作 注意到了这一点，但是没有从不同类型的关系出 发讨论环状结构的成因 ${ }^{[11]}$. 下面重点阐述这一点.

环状结构的图谱可以分为 3 类: (1) 对称关系 形成的环，其中头部实体和尾部实体互换，三元组 仍然成立; (2) 互逆关系形成的环，即 2 个不同关 系的头/尾部实体互为另一个关系的尾/头部实体; (3) 关系路径形成的环，即不同关系的复合. 在这 3
类情况下，不同实体、关系具备相同表示或表示为 零向量的现象, 都会对推理过程带来严重的干扰, 本文称为“退化”现象.

究其根源, 在于平移距离模型忽略了实体在 头部和尾部所带来的语义差异. 为此, 本文提出位 置敏感的嵌人模型, 希望成立的约束 $f_{r}(\boldsymbol{h})=\boldsymbol{t}$, 即 关系对实体的作用仅施加于头部实体，而保留尾 部实体的原始语义. 同时, 关系对头部实体的语义 变换不再局限于平移，而是一般的线性变换.

针对知识图谱嵌人存在的问题, 本文提出位 置敏感的距离模型. 理论分析和实验结果都说明 了本文模型可以达到当前最先进的性能. 在新模 型中, 进一步对关系的变换进行简化, 可以达到线 性时间复杂度，使模型可以拓展到大规模知识图 谱上. 本文同时改进了负采样的概率分布, 避免在 模型训练过程中出现大量无意义的负样本. 实验 证明，该技巧能提高模型的最终性能。

\section{1 相关工作}

在介绍相关工作之前, 简单介绍本文的符号 系统. 使用行向量 $\boldsymbol{h}, \boldsymbol{r}, \boldsymbol{t} \in \mathbb{R}^{d}$ 表示符号化的三元组 $(h, r, t)$ (在有的工作中, 关系和实体的维数可以 不同, 为求简洁, 本文采用相同维数); 使用 $s(h, r, t)$ 表示对三元组成立概率的评分.

知识图谱嵌人通常采用排序损失作为优化目 标，即 $\min \mathcal{L}=\sum_{(h, r, t)} \sum_{\left(h^{\prime}, r, t^{\prime}\right)}\left[\gamma+s(h, r, t)-s\left(h^{\prime}, r, t^{\prime}\right)\right]_{+}$. 其中, $[x]_{+}=\max (0, x) ; \gamma$ 控制正负样本距离. 由 于知识图谱不收录负样本, 因此 $\left(h^{\prime}, r, t^{\prime}\right)$ 是随机替 换正确三元组 $(h, r, t)$ 的头部实体或尾部实体(但不 能同时替换二者)所得到的. 这个过程称为负采样. 负采样的过程中可能会引人假阴性三元组, 即正 确成立只是未收录的三元组, 后文对这点再进行 讨论.

也可以采用交叉熵损失作为优化目标，即

$$
\min \mathcal{L}=-\sum_{(h, r, t)} \ln p(h, r, t)-\sum_{\left(h^{\prime}, r, t^{\prime}\right)} \ln \left(1-p\left(h^{\prime}, r, t^{\prime}\right)\right) .
$$

在这种情况下，距离评分经过 Sigmoid 函数后 得到成立的概率，即 $p(h, r, t)=\sigma(\gamma-s(h, r, t))$.

已有研究表明 ${ }^{[3]}$, 对于平移距离模型, 排序损 
失效果更好; 对于语义匹配模型，交叉熵损失效果 更好. 另外, 知识图谱嵌人通常对实体和向量的长 度有限制 ${ }^{[3]}$, 本文取消了这些限制条件.

\section{1 平移距离模型}

平移距离模型将关系建模为平移变换, 头部 实体经过平移之后和尾部实体之间的距离越小, 则三元组成立的可能性越大. 从统一框架的视角 来看, TransE 及其改进模型都是距离 $\left\|\boldsymbol{h} \boldsymbol{R}_{r}+\boldsymbol{r}-\boldsymbol{t} \boldsymbol{R}_{r}\right\|_{p}$ 的特例. 其中, $\boldsymbol{R}_{r}$ 是由各个关系决定的; $p=1,2$ 为 1 范数或 2 范数.

TransE $E^{[5]}$ 将距离评分定义为 $\|\boldsymbol{h}+\boldsymbol{r}-\boldsymbol{t}\|_{p}$. 可见, 如果 $\boldsymbol{R}_{r}=\boldsymbol{I}$, 即为 TransE 模型.

在平移变换之前, $\mathrm{TransR}^{[6]}$ 先将实体映射到不 同关系决定的空间中，以便捕捉实体在不同关系 链接中的不同语义, 即 $\left\|\boldsymbol{h} \boldsymbol{R}_{r}+\boldsymbol{r}-\boldsymbol{t} \boldsymbol{R}_{r}\right\|_{p}$.

$\mathrm{TransH}^{[7]}$ 进一步假设映射矩阵进行了投影变 换，投影平面由各个关系所决定. 记投影面的单位 法向量为 $\boldsymbol{w}_{r}$, 则投影为 $\boldsymbol{e}-\boldsymbol{e} \boldsymbol{w}_{r}^{\mathrm{T}} \boldsymbol{w}_{r}$.

$\mathrm{TransD}^{[9]}$ 假设映射矩阵由关系及其连接的实 体共同决定, 并将矩阵分解成为 2 个向量的乘积, 即 $\boldsymbol{R}_{r}=\boldsymbol{w}_{r}^{\mathrm{T}} \boldsymbol{w}_{e}+\boldsymbol{I}$.

$\operatorname{TransA}^{[8]}$ 采用 $(|\boldsymbol{h}+\boldsymbol{r}-\boldsymbol{t}|) \boldsymbol{M}_{r}(|\boldsymbol{h}+\boldsymbol{r}-\boldsymbol{t}|)^{\mathrm{T}}$, 其中 假设 $\boldsymbol{M}_{r}$ 对称. 下面说明它是 $\left\|\boldsymbol{h} \boldsymbol{R}_{r}+\boldsymbol{r}-\boldsymbol{t} \boldsymbol{R}_{r}\right\|_{p}$ 的一 个特例. 假设 $\left\|\boldsymbol{h} \boldsymbol{R}_{r}+\boldsymbol{r}-\boldsymbol{t} \boldsymbol{R}_{r}\right\|_{p}$ 中的映射矩阵可逆, 即 $\boldsymbol{R}_{r}^{-1}$ 存在，则其可改写为 $\left\|\left(\boldsymbol{h}+\boldsymbol{r}^{\prime}-\boldsymbol{t}\right) \boldsymbol{R}_{r}\right\|_{p}$; 其中, $\boldsymbol{r}^{\prime}=\boldsymbol{r} \boldsymbol{R}_{r}^{-1}$. 当 $p=2$, 即为 $\left(\boldsymbol{h}+\boldsymbol{r}^{\prime}-\boldsymbol{t}\right) \boldsymbol{R}_{r} \boldsymbol{R}_{r}^{\mathrm{T}}\left(\boldsymbol{h}+\boldsymbol{r}^{\prime}\right.$ $-\boldsymbol{t})^{\mathrm{T}}$. 记 $\boldsymbol{M}_{r}=\boldsymbol{R}_{r} \boldsymbol{R}_{r}^{\mathrm{T}}$ 为半正定矩阵, 则可以省略取 绝对值的操作, 即为 TransA 模型.

此外, TranSparse ${ }^{[12]}$ 考虑了稀疏的映射矩阵, $\operatorname{TransM}^{[13]}$ 采用 $\theta_{r}\left\|\boldsymbol{h} \boldsymbol{R}_{r}+\boldsymbol{r}-\boldsymbol{t} \boldsymbol{R}_{r}\right\|_{p}$, 通过调节参数放 松对部分三元组的限制. 这些模型都是一些特例 而已.

\section{2 语义匹配模型}

这类模型采用相似度评分，不同模型的区别 主要在于如何捕捉嵌人表示之间的相互影响.

$\operatorname{RESCAL}^{[14]}$ 采用二次型评分函数，即 $\boldsymbol{h} \boldsymbol{M}_{r} \boldsymbol{t}^{\mathrm{T}}$, 捕捉实体的潜在因子两两之间的相互影响; Dist$\mathrm{Mult}^{[15]}$ 进一步将矩阵简化为对角阵, 采用 $\boldsymbol{h} \operatorname{diag}(\boldsymbol{r}) \boldsymbol{t}^{\mathrm{T}}$ ，这种过度简化使模型不能区分对称关 系. ComplEx ${ }^{[16]}$ 研究了点乘操作对反对称关系的建 模瓶颈，并提出将 DistMult 拓展到复数空间中，即
评分函数为 $\operatorname{Re}\left(\boldsymbol{h} \operatorname{diag}(\boldsymbol{r}) \overline{\boldsymbol{t}}^{\mathrm{T}}\right)$. $\mathrm{HolE}^{[17]}$ 采用循环相关 算子聚合头部实体和尾部实体的相互影响, 即 $(\boldsymbol{h} * \boldsymbol{t}) \boldsymbol{r}^{\mathrm{T}}$; 其中, $[\boldsymbol{h} * \boldsymbol{t}]_{i}=\sum_{k=0}^{d-1}[\boldsymbol{h}]_{k} \cdot[\boldsymbol{t}]_{(k+i) \bmod d} \cdot$ 可 以证明，对于任一 ComplEx 模型，均存在等价的 $\mathrm{HolE}^{[18]}$.

此外，语义匹配模型有一个分支是基于神经 网络的，包括语义匹配嵌人 (semantic matching embedding, SME) ${ }^{[19]}$ 、神经张量网络(neural tensor networks, NTN $)^{[20]}$ 等. 在这个分支中，卷积嵌人模 型(convolutional embedding, ConvE) ${ }^{[21]}$ 是性能最好 的模型; 它将头部实体和关系的向量进行重组和 堆叠, 然后使用卷积层进行特征提取, 最后与尾部 实体一起通过全连接层进行匹配. 由于采用大量 卷积，其计算复杂度较高.

\section{2 位置敏感的距离模型}

本节首先分析了平移距离模型不能建模的几 类关系; 其次, 提出了位置敏感的改进距离模型, 同时，本文不再将关系建模为平移，而是一般的线 性变换, 并从理论上分析其具有较好的表示能力; 随后，提出了简化模型; 最后, 讨论了模型和其他 现有模型之间的关系和区别.

\section{1 平移距离模型失效的原因}

记知识图谱 $G=\{E, R\}$, 其中, $E$ 代表全部实 体的集合, 数量记为 $n_{e}, R$ 表示全体关系的集合, 数量记为 $n_{r}$. 本文总结 3 种特定模式的关系: 对称 性、互逆性和组合性，并给出具体定义.

定义 1. 如果 $\forall a, b \in E,(a, r, b) \rightarrow(b, r, a)$, 称 关系 $r$ 对称，如图 1a 所示.

定义 2. 如果 $\forall a, b \in E,\left(a, r_{1}, b\right) \rightarrow\left(b, r_{2}, a\right)$ ， 称关系 $r_{1}$ 和 $r_{2}$ 互逆, 如图 $1 \mathrm{~b}$ 所示.

定义 3. 如果 $\forall a, b, c \in E, \quad\left(a, r_{1}, b\right) \wedge\left(b, r_{2}, c\right) \rightarrow$ $(a, r, c)$, 称关系 $r$ 是 $r_{1}$ 和 $r_{2}$ 的组合, 如图 1c 所示.

如前所述, 这 3 种关系带来的环状结构使平移 距离模型产生退化现象. (1) 对称关系要求 $f_{r}(a)+$ $r=f_{r}(b)$ 与 $f_{r}(b)+r=f_{r}(a)$ 同时成立, 这导致 $r=0$; 更进一步，通常 $f_{r}($.$) 是可逆线性变换，则导致$ $a=b$ ；（2）互逆关系要求 $f_{r_{1}}(a)+r_{1}=f_{r_{1}}(b)$ 与 $f_{r_{2}}(a)+r_{2}=f_{r_{2}}(b)$ 同时成立, 存在非平凡的解满足 这个条件, 因此平移距离模型可以建模互逆关系; (3) 组合关系要求 $f_{r_{1}}(a)+r_{1}=f_{r_{1}}(b), f_{r_{2}}(b)+r_{2}=f_{r_{2}}(c)$ 与 


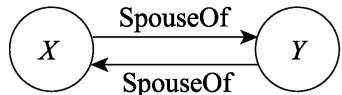

a. 对称性

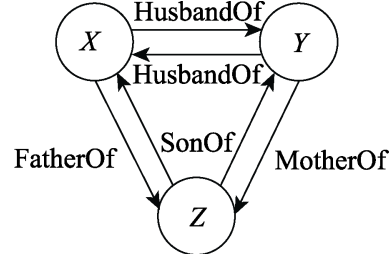

b. 互逆性

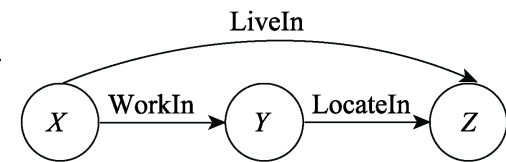

c. 组合性

图 13 种特殊类型关系构成的知识图谱

$f_{r_{3}}(a)+r_{3}=f_{r_{3}}(c)$ 同时成立，将导致 $f_{r_{3}}(\cdot)=f_{r_{2}}(\cdot)$. 后者表示对不同关系采用了相同的映射函数，这 是 TransE 的改进模型(包括 TransR 等)并没有实际 超过 TransE 的重要原因.

通过上述推导可以看出，问题根源在于对三 元组的头部实体和尾部实体采用了相同的映射. 因此, 本文提出一种位置敏感的改进距离模型.

\section{2 位置敏感的改进距离模型}

最直观的想法是, 对三元组的头部实体保留 线性变换, 但是对尾部实体则不做任何变换, 即期 望模型对于正确的三元组成立关系 $f_{r}(\boldsymbol{h})+\boldsymbol{r}=\boldsymbol{t}$. 由 于平移算子可以看成线性变换的一部分，即可以 将其作用归人 $f_{r}(\cdot)$ 之中. 最终, 本文提出位置敏 感的距离模型(location-sensitive embedding, LSE), 三元组距离评分函数为 $\left\|\boldsymbol{h} \boldsymbol{R}_{r}-\boldsymbol{t}\right\|_{p}$. 同样地, 距离越 小, 该三元组成立的可能性越高. $p$ 可以取 1 范数 或 2 范数, 通过实验, 本文选取 1 范数效果更佳.

下述给出 3 个引理，它们说明了 LSE 模型能够 建模前述 3 类特定模式的关系.

引理 1. 改进距离模型可以建模对称关系.

证明. 根据 $\boldsymbol{R}_{r} \boldsymbol{a}=\boldsymbol{b}, \boldsymbol{R}_{r} \boldsymbol{b}=\boldsymbol{a}$, 可得 $\boldsymbol{R}_{r} \boldsymbol{R}_{r}=\boldsymbol{I}$. 满足这一条件不会对实体嵌人有额外限制. 证毕.

引理 2. 改进距离模型可以建模互逆关系.

证明. 根据 $\boldsymbol{R}_{r_{1}} \boldsymbol{a}=\boldsymbol{b}, \boldsymbol{R}_{r_{2}} \boldsymbol{b}=\boldsymbol{a}$, 可得 $\boldsymbol{R}_{r_{1}} \boldsymbol{R}_{r_{2}}=\boldsymbol{I}$. 满足这一条件不会对实体嵌人有额外限制. 证毕.

引理 3. 改进距离模型可以建模关系的组合.

证明. 根据 $\boldsymbol{R}_{r_{1}} \boldsymbol{a}=\boldsymbol{b}, \boldsymbol{R}_{r_{2}} \boldsymbol{b}=\boldsymbol{c}, \boldsymbol{R}_{r_{3}} \boldsymbol{a}=\boldsymbol{c}$, 可得 $\boldsymbol{R}_{r_{2}} \boldsymbol{R}_{r_{1}}=\boldsymbol{R}_{r_{3}}$, 满足这一条件不会对实体嵌人有额外 限制.

证毕.

\subsection{1 损失函数}

为了更好地考虑未观测样本中可能成立的三 元组，本文实验了带标签平滑 (label-smooth)的交 叉熵损失 (cross-entropy). 对于知识图谱中已收录 的三元组, 其标签仍然是 1 ; 对于随机负采样得到 的 $k$ 组样本, 其标签不是 0 , 而是 $1 / k$; 其中, $k>1$ 是 对每个三元组负采样的比例. 损失函数为

$$
\begin{aligned}
& \min \mathcal{L}=- \\
& \quad \sum_{(h, r, t)}\left\{\log p(h, r, t)-\sum_{\left(h^{\prime}, r, t^{\prime}\right)} \frac{1}{k} \log \left(1-p\left(h^{\prime}, r, t^{\prime}\right)\right)\right\} .
\end{aligned}
$$

注意, 本文移除了所有对参数的范数的限制, 因此损失函数没有正则项.

\subsection{2 模型的变式}

在 LSE 模型中, 采用矩阵表示映射函数会造 成计算量较大. 为解决此问题, 本文同时假设该矩 阵可以用对角阵替代, 即对头部实体的映射操作, 可以在各维度上独立地进行. 本文称模型为 $\mathrm{LSE}_{d}$, 它使用距离评分函数 $\|\boldsymbol{h} \operatorname{diag}(\boldsymbol{r})-\boldsymbol{t}\|_{p}=\|\boldsymbol{h} \circ \boldsymbol{r}-\boldsymbol{t}\|_{p}$. 注 意到 $\|\boldsymbol{h} \circ \boldsymbol{r}-\boldsymbol{t}\|_{1}=\sum_{i=1}^{d}\left|h_{i} \cdot r_{i}-t_{i}\right|$, 等式右边累加项可以 看做一维的嵌人模型. 由于关系对实体的变换是 各个维度独立进行的, 因此 $\mathrm{LSE}_{d}$ 可以看成是集成 了 $d$ 个一维的嵌人模型.

\subsection{3 与其他模型的比较与联系}

在现有的知识图谱嵌人模型中, 平移距离模 型和本文模型关系最为密切. 除此之外, 无结构模 型 (unstructured model, UM) ${ }^{[22]}$ 和结构化嵌人 (structured embedding, SE) ${ }^{[23]}$ 与 LSE 相同, 均不采 用平移算子. $\mathrm{UM}$ 的距离评分函数为 $\|\boldsymbol{h}-\boldsymbol{t}\|_{2}, \mathrm{SE}$ 则 采用 $\left\|\boldsymbol{R}_{r}^{1} \boldsymbol{h}-\boldsymbol{R}_{r}^{2} \boldsymbol{t}\right\|_{2}$. 前者过度简化了关系的作用, 后者虽然采用了和本文类似的想法，但是引人了 过多的参数, 不利于模型的训练.

除此之外, RESCAL ${ }^{[15]}$ 与 DistMult ${ }^{[16]}$ 都可以看 成也对头部实体进行了线性变换. 不同的是, 它们 采用内积计算相似度. 如果限制本文模型中的向 量变换前后的均为单位模长, 且令 $p=2$, 则 $\left\|\boldsymbol{h} \boldsymbol{R}_{r}-\boldsymbol{t}\right\|_{2}^{2}=2-2 \boldsymbol{h} \boldsymbol{R}_{r} \boldsymbol{t}^{\mathrm{T}}$ ，与 RESCAL 等价. 可见, 较 RESCAL 而言, 本文模型移除了模长的限制, 因此 具有更好的表示能力. 在 DistMult 中, 交换头部实 体和尾部实体得到的评分是一样的, 这将导致模 型将所有关系都视为对称关系. 本文提出的简化 模型 $\mathrm{LSE}_{d}$ 规避了这一点.

表 1 列举了与当前先进模型复杂度的比较结 
果. 可以看出, $\mathrm{LSE}_{d}$ 作为线性时间复杂度模型, 可 以应用于大规模知识图谱的推理任务中.

\section{表 1 知识图谱嵌入模型复杂度比较}

\begin{tabular}{lll}
\hline \multicolumn{1}{c}{ 模型 } & \multicolumn{1}{c}{ 空间复杂度 } & 时间复杂度 \\
\hline $\operatorname{TransE}^{[5]}$ & $\left(n_{r}+n_{e}\right) d$ & $O(d)$ \\
$\operatorname{TransR}^{[6]}$ & $\left(n_{r}+n_{e}\right) d+n_{r} d^{2}$ & $O\left(d^{2}\right)$ \\
$\operatorname{DistMult}^{[15]}$ & $\left(n_{r}+n_{e}\right) d$ & $O(d)$ \\
$\mathrm{HolE}^{[17]}$ & $\left(n_{r}+n_{e}\right) d$ & $O(d \ln d)$ \\
$\mathrm{ComplEx}^{[16]}$ & $2\left(n_{r}+n_{e}\right) d$ & $O(d)$ \\
$\mathrm{ConvE}^{[21]}$ & $\left(n_{r}+n_{e}\right) d$ & \\
\hline $\mathrm{LSE}_{\mathrm{LSE}}$ & $n_{e} d+n_{r} d^{2}$ & $O\left(d^{2}\right)$ \\
& $\left(n_{r}+n_{e}\right) d$ & $O(d)$ \\
\hline
\end{tabular}

\section{3 实验结果与讨论}

本节首先介绍了实验使用的数据集、知识图谱 的链接预测任务、评价指标等设置, 然后讨论超参 数的影响和选取技巧, 最后列举了改进距离模型 的性能, 并与当前性能最好的模型进行对比分析.

值得一提的是, 已有的研究工作表明, 许多知 识图谱嵌人方法(包括 TransE, DistMult 等)经过精 细调参, 均能超过它们原始公布的结果. 这也是平 移距离模型的改进工作一直受到诟病的一点, 即 对比方法原文未经过精细调参，但事实上一些基 准方法仍有较大的训练提升空间. 为了保证对比 的公平性, 本文选择了文献[10,21,24-25]等研究工 作作为对比方法的出处.

\section{1 实验设置}

\section{1 .1 数据集}

在 4 个公开数据集上评测本文模型, 它们的统 计特性如表 2 所示.

表 2 数据集实体、关系、三元组的数量及切分

\begin{tabular}{lrcrcr}
\hline \multicolumn{1}{c}{ 数据集 } & \multicolumn{1}{c}{$n_{r}$} & $n_{e}$ & $\begin{array}{c}\text { 训练集 } \\
\text { 数量 }\end{array}$ & $\begin{array}{c}\text { 验证集 } \\
\text { 数量 }\end{array}$ & $\begin{array}{c}\text { 测试集 } \\
\text { 数量 }\end{array}$ \\
\hline FB15k & 1345 & 14951 & 483142 & 50000 & 59071 \\
FB15k-237 & 237 & 14541 & 272115 & 17535 & 20466 \\
WN18 & 18 & 40943 & 141442 & 5000 & 5000 \\
WN18RR & 11 & 40943 & 86835 & 3034 & 3134 \\
\hline
\end{tabular}

(1) FB15k 与 FB15k-237. FB15k 是事实数据库 Freebas ${ }^{[26]}$ 的子集. 该数据库主要的缺点在于, 测 试集中 $81 \%$ 的三元组可以通过逆关系推理得出. 这些关系在 FB15k-237 中都删掉了, 因此变得更具 挑战性.

(2) WN18 与 WN18RR. WN18 是单词层次关系 数据库 WordNet ${ }^{[27]}$ 的子集. 相比于 Freebase,
WordNet 的实体数量更多, 关系类型更少, 同时形 成的三元组数量也更少, 这说明平均每个实体节 点的相互连接是比较稀疏的, 给知识图谱表示学 习带来了较大的难题. WN18 同样存在很多可逆的 关系, WN18RR 删除了这些关系.

\subsection{2 链接预测与评价指标}

链接预测本质是一个排序问题. 在测试阶段, 对于每一个三元组, 使用图谱中全体实体替换其 头部或尾部实体, 然后对所有的替换结果的距离 评分进行升序排列. 对排序结果，本文使用 2 类指 标进行评价.

(1) 平均排行倒数 (mean reciprocal rank, MRR). 正确实体在所有实体中排行的倒数求平均, 即 $\sum_{i=1}^{N}\left(1 / \operatorname{rank}_{i}\right) / N$. 正确实体排名越靠前, MRR 越高.

(2) 前 $n$ 命中率(Hits@n). 正确实体出现在排 名前 $n$ 的实体中的比例. 本文分别统计 $n=1,3,10$ 时的情况.

有的文献也使用平均排行 (mean rank) 作为评 价指标, 但是其受到极端样本的影响太大, 不如 MRR 较为稳定、客观. 另外, 替换后产生的三元组 并不一定是负样本, 其本身可能也存在知识图谱 之中. 上述计算方式可能会低估了模型的性能. 为 了得到公平的评价，一般采用“过滤”的设置，即在 计算评价指标之前, 先将正确的三元组从排序结 果中过滤掉.

\section{2 实验环境与超参数}

本文代码基于 PyTorch 框架，使用单块 Titan X GPU(12 GB)进行训练.

本文所提出的 LSE 和 $\mathrm{LSE}_{d}$ 模型涉及的超参数 包括 3 类: (1) 模型超参数. 嵌人维数 $d \in\{100,200$, $500,1000\}$, 正负样本的间隔 $\gamma \in\{1,2, \cdots, 30\}$. (2) 优化算法超参数. 模型训练采用随机梯度下降 (stochastic gradient descent, SGD)算法, 其涉及的 超参数及其选取范围为学习率 $l \in\left\{5 \times 10^{-4}, 10^{-4}\right.$, $\left.5 \times 10^{-5}, 10^{-5}\right\}$ ，批数据大小为 $b \in\{128,256,512$, $1024\}$, 迭代次数 $p \in\left\{5 \times 10^{4}, 6 \times 10^{4}, \cdots, 10^{5}\right\}$. (3) 负采样超参数. 本文根据 $\mathrm{Trans}^{[7]}$ 办法, 采用伯努 利分布随机替换头部实体或尾部实体，避免均匀 采样的过程中引人过多的假阴性(false negative)三 元组. 对于每一对三元组, 随机负采样的比例为 $g \in\{128,256,512,1024\}$.

通过验证集的选择，本文对 4 个数据集都使用 $d=500$. 对于 FB15k, $\gamma=24, l=5 \times 10^{-4}, b=256$, $p=10^{5}, g=256$; 对于 FB15k-237, $\gamma=9, l=5 \times 10^{-4}$, 
$b=1024, p=10^{5}, g=256$; 对于 WN18, $\gamma=12, l=$ $5 \times 10^{-4}, b=512, p=5 \times 10^{4}, g=1024$; 对于 WN18RR, $\gamma=6, l=5 \times 10^{-4}, b=512, p=10^{5}, g=1024$. 本文在 参数调节过程中发现，在实验显存允许的条件下， 扩大批数据大小, 增加负采样比例, 都会带来性能 上的提高.

\section{3 实验结果与分析}

表 3 和表 4 分别比较了 $\mathrm{LSE}, \mathrm{LSE}_{d}$ 模型与其他
对比模型在链接预测任务上的性能. 可以看到, 在 FB15k-237 和 WN18RR 数据集上, 本文提出的模 型均达到了最佳的性能; 在 FB15k 和 WN18 数据 集上, 本文提出的模型也与最佳模型保持了基本 持平的性能. 另外, $\mathrm{LSE}_{d}$ 相比于 LSE 有大幅度的 性能提升, 这说明较少的参数反而更加适合训练, 也说明了本文对于各个维度相互独立的假设是成 立的.

表 3 改进距离模型在 FB15k 和 WN18 数据集上的链接预测性能

\begin{tabular}{|c|c|c|c|c|c|c|c|c|}
\hline \multirow{2}{*}{ 模型 } & \multicolumn{4}{|c|}{ FB15k } & \multicolumn{4}{|c|}{ WN18 } \\
\hline & MRR & Hits@1 & Hits@3 & Hits@10 & MRR & Hits@1 & Hits@3 & Hits@10 \\
\hline TransE $E^{[10]}$ & 0.463 & 0.297 & 0.578 & 0.749 & 0.495 & 0.113 & 0.888 & 0.943 \\
\hline DistMult $^{[24]}$ & 0.798 & & & 0.893 & 0.797 & & & 0.946 \\
\hline $\mathrm{HolE}^{[17]}$ & 0.524 & 0.402 & 0.613 & 0.739 & 0.938 & 0.930 & 0.945 & 0.949 \\
\hline ComplEx ${ }^{[16]}$ & 0.692 & 0.599 & 0.759 & 0.840 & 0.941 & 0.936 & 0.945 & 0.947 \\
\hline ConvE $^{[21]}$ & 0.745 & 0.670 & 0.801 & 0.873 & 0.943 & 0.935 & 0.946 & 0.956 \\
\hline LSE & 0.701 & 0.643 & 0.733 & 0.795 & 0.873 & 0.842 & 0.898 & 0.926 \\
\hline $\mathrm{LSE}_{d}$ & 0.742 & 0.680 & 0.783 & 0.848 & 0.929 & 0.910 & 0.944 & 0.955 \\
\hline
\end{tabular}

注. 粗体表示最佳性能.

表 4 改进距离模型在 FB15k-237 和 WN18RR 数据集上的链接预测性能

\begin{tabular}{|c|c|c|c|c|c|c|c|c|}
\hline \multirow{2}{*}{ 模型 } & \multicolumn{4}{|c|}{ FB15k-237 } & \multicolumn{4}{|c|}{ WN18RR } \\
\hline & MRR & Hits@1 & Hits@3 & Hits@10 & MRR & Hits@1 & Hits@3 & Hits@10 \\
\hline TransE $E^{[10]}$ & 0.294 & & & 0.465 & 0.226 & & & 0.501 \\
\hline DistMult $^{[24]}$ & 0.241 & 0.155 & 0.263 & 0.419 & 0.430 & 0.390 & 0.440 & 0.490 \\
\hline ComplEx ${ }^{[16]}$ & 0.247 & 0.158 & 0.275 & 0.428 & 0.440 & 0.410 & 0.460 & 0.510 \\
\hline ConvE $E^{[21]}$ & 0.325 & 0.237 & 0.356 & 0.501 & 0.430 & 0.400 & 0.440 & 0.520 \\
\hline LSE & 0.299 & 0.189 & 0.312 & 0.470 & 0.421 & 0.378 & 0.450 & 0.494 \\
\hline $\mathrm{LSE}_{d}$ & 0.331 & 0.237 & 0.366 & 0.522 & 0.450 & 0.407 & 0.465 & 0.537 \\
\hline
\end{tabular}

注. 粗体表示最佳性能.

具体到各个数据集上, 有以下发现.

(1) 在 FB15k 数据集上, DistMult 能够得到最 高的性能. 这说明在这个数据集中, 对称关系是主 要的关系模式. 事实上, 大约 $81 \%$ 测试集三元组可 以通过关系的对称性直接推理得到 ${ }^{[4]}$. 可以看到, 在其他的数据集上, DistMult 的效果远不如本文提 出的模型, 因此可以说, 本文提出的模型比 DistMult 更加通用.

(2) 在 WN18 数据集上, 主要的关系模式是对 称和互逆; WN18RR 数据集上则主要是对称(如关 系 also see, similar to 等). 在这 2 个数据集上, TransE 的性能均明显地下降, 说明其不能很好地 对称关系. 这与第 3 节的理论分析是一致的.

(3) 在 FB15k-237 和 WN18RR 数据集上, 关系 的复合也占据着很重要的部分. 在 FB15k-237 中, TransE 的效果和其他先进的算法并没有太大的差 别, 这与第 3 节的理论分析是一致的. 同样地, 本
文所提出的模型也能很好地建模关系的组合.

综合来看, 本文实验结果与理论分析对应关 系很好, 充分说明了本文模型 LSE 和 $\mathrm{LSE}_{d}$ 具有良 好的知识图谱表示学习能力.

\section{4 结 语}

本文提出了一种用于知识图谱嵌人表示的位 置敏感模型, 从理论和实验验证了所提出的改进 模型的有效性, 并在大规模知识图谱的链接预测 任务上取得了优越的性能.

本文注意到仍然有一些问题需要进一步的研 究. (1) 在组合关系中, 有一类特殊情况需要单独 考虑, 即由相同关系形成的组合, 这种模式通常存 在于层次结构较多的专业领域知识图谱中. 例如, (小臂, partOf，四肢)和(四肢, partOf，人体), 同时 存在(小臂, partOf, 人体) 的知识关系. 目前的模 
型不能很好地处理这种关系. (2) 知识的表示具有 一定的不确定性，在 $\mathrm{KG} 2 \mathrm{E}^{[28]}$ 模型中，实体和关系 不再被建模为空间中的一个点，而是一个高斯分 布. 本文所提出的位置敏感嵌人在不确定性知识 表示的应用方面还需要进一步完善.

\section{参考文献(References):}

[1] Singhal A. Introducing the knowledge graph: things, not strings[OL]. [2020-08-06]. https://blog.google/products/search/ introducing-knowledge-graph-things-not/

[2] Nickel M, Murphy K, Tresp V, et al. A review of relational machine learning for knowledge graphs[J]. Proceedings of the IEEE, 2016, 104(1): 11-33

[3] Wang Q, Mao Z D, Wang B, et al. Knowledge graph embedding: a survey of approaches and applications[J]. IEEE Transactions on Knowledge and Data Engineering, 2017, 29(12): 2724-2743

[4] Toutanova K, Chen D Q. Observed versus latent features for knowledge base and text inference[C] //Proceedings of the 3rd Workshop on Continuous Vector Space Models and their Compositionality. Cambridge: MIT Press, 2015: 57-66

[5] Bordes A, Usunier N N, Garcia-Duran A. Translating embeddings for modeling multi-relational data[C] //Proceedings of the 29th International Conference on Neural Information Processing Systems. Cambridge: MIT Press, 2013: 2787-2795

[6] Lin Y K, Liu Z Y, Sun M S, et al. Learning entity and relation embeddings for knowledge graph completion[C] //Proceedings of the 29th AAAI Conference on Artificial Intelligence. Palo Alto: AAAI Press, 2015: 2181-2187

[7] Wang Z, Zhang J W, Feng J L, et al. Knowledge graph embedding by translating on hyperplanes[C] //Proceedings of the 28th AAAI Conference on Artificial Intelligence. Palo Alto: AAAI Press, 2014: 1112-1119

[8] Xiao H, Huang M L, Yu H, et al. TransA: an adaptive approach for knowledge graph embedding[OL]. [2020-08-06]. https:// arxiv.org/pdf/1509.05490.pdf

[9] Ji G L, He S Z, Xu L H, et al. Knowledge graph embedding via dynamic mapping matrix $[\mathrm{C}] / /$ Proceedings of the 53rd Annual Meeting of the Association for Computational Linguistics and the 7th International Joint Conference on Natural Language Processing. Cambridge: MIT Press, 2015: 687-696

[10] Akrami F, Guo L B, Hu W, et al. Re-evaluating embedding-based knowledge graph completion methods[C] //Proceedings of the 27th ACM International Conference on Information and Knowledge Management. New York: ACM Press, 2018: 1779-1782

[11] Zhang W. Knowledge graph embedding with diversity of structures[C] //Proceedings of the 26th International Conference on World Wide Web Companion. Switzerland: International World Wide Web Conferences Steering Committee Press, 2017: 747-753

[12] Ji G L, Liu K, He S Z, et al. Knowledge graph completion with adaptive sparse transfer matrix[C] //Proceedings of the 30th AAAI Conference on Artificial Intelligence. Palo Alto: AAAI Press, 2016: 985-991

[13] Fan M, Zhou Q, Chang E, et al. Transition-based knowledge graph embedding with relational mapping properties[C] //Pro- ceedings of the 28th Pacific Asia Conference on Language, Information and Computing. Bangkok: Department of Linguistics, Chulalongkorn University, 2014: 328-337

[14] Nickel M, Tresp V, Kriegel H P. A three-way model for collective learning on multi-relational data[C] //Proceedings of the 28th International Conference on Machine Learning. Madison: Omni Press, 2011: 809-816

[15] Yang B S, Yih W T, He X D, et al. Embedding entities and relations for learning and inference in knowledge bases[C] //Proceedings of the International Conference on Learning Representations. Cambridge: MIT Press, 2015: 51-63

[16] Trouillon T, Welbl J, Riedel S, et al. Complex embeddings for simple link prediction[C] //Proceedings of the 33rd International Conference on Machine Learning. New York: ACM Press, 2016: 2071-2080

[17] Nickel M, Rosasco L, Poggio T. Holographic embeddings of knowledge graphs[C] //Proceedings of the 33th AAAI Conference on Artificial Intelligence. Palo Alto: AAAI Press, 2016: 1955-1961

[18] Hayashi K, Shimbo M. On the equivalence of holographic and complex embeddings for link prediction[C] //Proceedings of the 55th Annual Meeting of the Association for Computational Linguistics. Cambridge: MIT Press, 2017: 554-559

[19] Bordes A, Glorot X, Weston J, et al. A semantic matching energy function for learning with multi-relational data[J]. Machine Learning, 2014, 94(2): 233-259

[20] Socher R, Chen D, Manning C D, et al. Reasoning with neural tensor networks for knowledge base completion[C] //Proceedings of Advances in Neural Information Processing Systems. Cambridge: MIT Press, 2013: 926-934

[21] Dettmers T, Minervini P, Stenetorp P, et al. Convolutional 2D knowledge graph embeddings[C] //Proceedings of the 32nd AAAI Conference on Artificial Intelligence. Palo Alto: AAAI Press, 2018: 1811-1818

[22] Bordes A, Glorot X, Weston J, et al. Joint learning of words and meaning representations for open-text semantic parsing[C] //Proceedings of the 15th International Conference of Artificial Intelligence and Statistics. New York: ACM Press, 2012: 127-135

[23] Bordes A, Weston J, Collobert R, et al. Learning structured embeddings of knowledge bases[C] //Proceedings of the 25th AAAI Conference on Artificial Intelligence. Palo Alto: AAAI Press, 2011: 301-306

[24] Kadlec R, Bajgar O, Kleindienst J. Knowledge base completion: baselines strike back[OL]. [2020-08-06]. https://arxiv.org/ abs/1705.10744

[25] Nguyen D Q, Nguyen T D, Nguyen D Q, et al. A novel embedding model for knowledge base completion based on convolutional neural network[OL]. [2020-08-06]. https://arxiv.org/ $\mathrm{abs} / 1712.02121 \mathrm{v} 2$

[26] Bollacker K, Evans C, Paritosh P, et al. Freebase: a collaboratively created graph database for structuring human knowledge[C] //Proceedings of the ACM SIGMOD International Conference on Management of Data. New York: ACM Press, 2008: $1247-1250$

[27] Miller G A. WordNet: a lexical database for English[J]. Communications of the ACM, 1995, 38(11): 39-41

[28] He S Z, Liu K, Ji G L, et al. Learning to represent knowledge graphs with Gaussian embedding[C] //Proceedings of the 24th ACM International on Conference on Information and Knowledge Management. New York: ACM Press, 2015: 623-632 